\title{
Unanticipated Difficult Intubation in Patient with Undiagnosed Posterior Glottic Stenosis
}

\author{
Seunghyeon Lee, Misoon Lee, Bon Sung Koo, Yang Hoon Chung \\ Department of Anesthesiology and Pain Medicine, Soonchunhyang University Bucheon Hospital, Bucheon, Korea
}

\begin{abstract}
Posterior glottic stenosis (PGS) is frequently caused by prolonged intubation complications, which may limit the movement of the vocal cords and obstruct airway obstruction. Despite of a life-threatening condition, it might be overlooked in asymptomatic individual. A 63-year-old female was scheduled for arthroscopic rotator cuff repair. The patient had experienced 2 times of intubation and organophosphate poisoning for suicide. Both of 7.0- and 6.5-mm inner diameter endotracheal tubes could not be passed vocal cords. After two failed attempts at intubation, adhesion of posterior part of vocal cord was revealed. We stopped the tracheal intubation and called the otolaryngologist, and adhesiolysis was performed under direct laryngosope. Thereafter, endotracheal intubation was performed successfully with 6.5-mm endotracheal tube.
\end{abstract}

Keywords: Difficult intubation; Posterior glottic stenosis; Airway management

\section{INTRODUCTION}

Posterior glottic stenosis (PGS) is frequent caused by complication of prolonged endotracheal intubation, which may limit the movement of the vocal cords and obstruct airway obstruction [1]. However, the diagnosis of PGS in patients without specific discomfort may be delayed, which leads to unexpected difficult intubation during general anesthesia because of the narrowed diameter of the airway. Here, we report a case of unexpected difficult intubation caused by undiagnosed PGS.

\section{CASE REPORT}

A 63-year-old female of $163 \mathrm{~cm}$ height and $67 \mathrm{~kg}$ weight with American Society of Anesthesiologists physical status classification III was scheduled for arthroscopic rotator cuff repair. Her medical history included hypertension, diabetes mellitus, allergic asthma, and coronary artery atherosclerosis. Before 11 years, she was admitted to intensive care unit due to organophosphate poisoning for suicide and underwent mechanical ventilation with endotracheal intubation. At that time, she was diagnosed with rhab- domyolysis, stress-induced cardiomyopathy, and pulmonary edema and was treated. She had a previous surgery for compression fracture of spine under general anesthesia without incident 20 years ago. Patient's airway assessment revealed Mallampati score I with a normal mouth opening and neck movements.

The patient was premedicated with glycopyrrolate $0.2 \mathrm{mg}$ intramuscular in the ward. On arrival of the patient to operation room, standard monitoring which consisted of electrocardiogram 3-lead continuous recording, noninvasive blood pressure measurement, capnography, and pulse oximetry was connected to the patient.

Ultrasound-guided interscalene brachial plexus block with 9 $\mathrm{mL}$ of $0.25 \%$ ropivacaine was performed prior to general anesthesia. After pre-oxygenation, general anesthesia was induced using propofol $80 \mathrm{mg}$ and fentanyl $50 \mu \mathrm{g}$. And then, after the loss of consciousness, rocuronium $50 \mathrm{mg}$ was administrated for muscle relaxation. Mask ventilation was easy. After verifying train-of-four count 0 measurement, endotracheal intubation was attempted with direct laryngoscope and plain 7.0-mm endotracheal tube. Most of vocal cords were exposed clearly, but endotracheal tube could not be passed vocal cords due to stenosis. The patient was ventilated again using a facial mask, and endotracheal intubation 


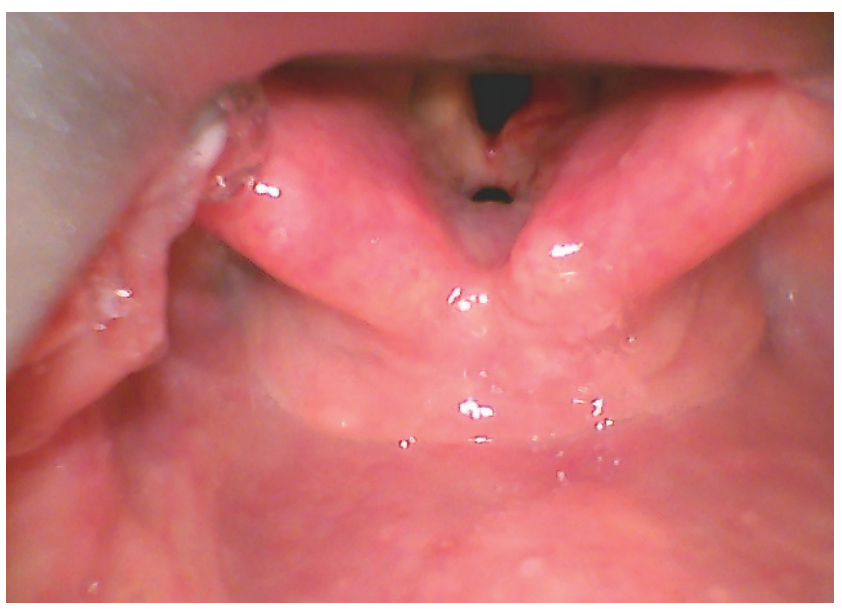

Fig. 1. Laryngoscopic finding: adhesion in posterior commissure of vocal cords.

was re-attempted using McGrath MAC (McGrath; Medtronic, Dublin, Ireland) video laryngoscope and smaller sized tube (6.5 $\mathrm{mm}$ ). However, this second attempt also failed to pass the vocal cords. After two failed attempts at intubation, adhesion of posterior part of vocal cord was clearly seen (Fig. 1). We stopped the endotracheal intubation and called the otolaryngologist immediately. At the same time, sevoflurane was discontinued and changed to total intravenous anesthesia using target controlled infusion (TCI) pump (Orchestra; Fresenius Vial, Brezins, France) at the affected site with propofol $6 \mu \mathrm{g} / \mathrm{mL}$ and remifentanil $3 \mathrm{ng} / \mathrm{mL}$. The otolaryngologist confirmed posterior commissure of vocal cords adhered to each other with a C-MAC Video Laryngoscope (Karl Storz GmbH \& Co. KG, Tuttlingen, Germany) and performed vocal cord adhesiolysis without remarkable problem. Thereafter, endotracheal intubation was performed successfully with $6.5-\mathrm{mm}$ endotracheal tube. After intubation, chest auscultation was normal. The endotracheal tube was secured at $21 \mathrm{~cm}$. Mechanical respiration was performed with a volume of $400 \mathrm{~mL}$ and a respiratory rate of 12 per minute. Total intravenous anesthesia using the TCI pump was maintained with propofol and remifentanil in air and oxygen. The surgery was uneventful.

The surgery was completed, and sugammadex (Bridion; Schering Plough, Istanbul, Turkey) $200 \mathrm{mg}$ was administrated intravenously. A few minutes later, T4/T1 reached 94\%. After verifying that the response entropy and state entropy increased up to 98 and 91, the patient was extubated uneventfully. The patient was transferred to the postoperative care unit, where she stayed for $54 \mathrm{~min}$ utes, and was then moved to ward without any immediate postoperative complication.

\section{DISCUSSION}

PGS is frequently caused by prolonged intubation complications, which may limit the movement of the vocal cords and obstruct airway obstruction. It is a life-threatening condition, especially in general anesthesia that endotracheal intubation is necessary. PGS is classified into four types according to Bogdassian and Olson [2], most commonly depending on the structure involved. Type I PGS (PGS-1) has epileptic fiber adhesion, patent opening, and posterior attachment. Type II-type IV progressively involve the additional structure of the posterior glottis [2]. Based on this classification, the patient was type I PGS.

The various causes of PGS include traumatic or long-term intubation, surgery, radiation, tumor invasion, systemic autoimmune disease, external trauma, and caustic ingestion. Of these various causes, complications of tracheal intubation are the most frequent causes. Injury to the interarytenoid mucosa and underlying cartilage resulting from tracheal intubation leads to interarytenoid fibrosis, contracture, and cricoarytenoid fixation of the joints. The pressure of the endotracheal tube causes mucosal necrosis, ulcers, local infection, and inflammation. After that, the process is fibrosis, and contracture and scarring are formed. The factors that increase the incidence of PGS are traumatic intubation, repeated intubation, long-term intubation, gastroesophageal reflux, largesized tube, tube movement, diabetes mellitus, congestive heart failure, and local infection $[2,3]$.

In this case, asymptomatic PGS caused unanticipated difficult intubation. However, despite the absence of specific symptoms, considering past history of 2 times of intubation and organophosphate poisoning, the possibility of difficult intubation should not be overlooked. Especially, the patient had medical history of 2 times of tracheal intubations and diabetes mellitus among the risk factors of PGS, and the intake of organic phosphate was also presumed to have influenced the onset of PGS [3].

The American Society of Anesthesiologists defined difficult intubation as three or more attempts using an average laryngoscope or 10 minutes or more. A guideline published in 2015 recommended the following procedure for unexpected difficult intubation: optimize head and neck postures, preoxygenate, adequate neuromuscular block, use of direct/videolaryngosopy $(3+1$ attempts), and maintain anesthesia and oxygen. If this first step fails, this guideline recommends using supraglottic airway devices (SAD) [4]. However, we did not consider the use of SAD in order to 
maintain stable airway because the surgery is scheduled to be performed in a side-lying position in this case.

In conclusion, a difficult tracheal intubation should always be aware of the situation that anesthesiologist can encounter at any time. In this reason, it is essential to understand a strategy for airway and prepare the necessary equipment. Above all, preoperative airway assessment is essential to identify patients at risk for difficult intubation.

\section{REFERENCES}

1. Meyer TK, Wolf J. Lysis of interarytenoid synechia (type I posterior glottic stenosis): vocal fold mobility and airway results. Laryngoscope 2011;121: 2165-71.

2. Bogdasarian RS, Olson NR. Posterior glottic laryngeal stenosis. Otolaryngol Head Neck Surg (1979) 1980;88:765-72.

3. Gelbard A, Francis DO, Sandulache VC, Simmons JC, Donovan DT, Ongkasuwan J. Causes and consequences of adult laryngotracheal stenosis. Laryngoscope 2015;125:1137-43.

4. Frerk C, Mitchell VS, McNarry AF, Mendonca C, Bhagrath R, Patel A, et al. Difficult Airway Society 2015 guidelines for management of unanticipated difficult intubation in adults. Br J Anaesth 2015;115:827-48. 